EPJ Web of Conferences [4, 01001 (2013)

DOI: $10.1051 /$ epjconf/20134601001

(C) Owned by the authors, published by EDP Sciences, 2013

\title{
Observations of inhomogeneities in protoplanetary disks
}

\author{
Cornelis Dullemond ${ }^{1, a}$ \\ ${ }^{1}$ Heidelberg University, Center for Astronomy, Institute for Theoretical Astrophysics, Albert Ueberle Str. 2, \\ 69120 Heidelberg, Germany
}

\begin{abstract}
In this review I will give a brief overview of the latest observational constraints on the structure and dynamics of protoplanetary disks, with a particular emphasis on the topic of the conference: vortices.
\end{abstract}

\section{Introduction}

The topic of this conference is vortices in protoplanetary disks. There are various theoretical arguments to support that such vortices should be present in protoplanetary disks, and if they do, they may play a crucial role in the process of planet formation. But $d o$ they indeed exist in protoplanetary disks? The only way to find out is to look for them in real protoplanetary disks: the dusty disks surrounding many T Tauri and Herbig Ae stars. This is, however, not an easy task. Most nearby young stars are located in the nearby molecular cloud complexes at distances of around 100 parsec. An entire protoplanetary disk of $50 \mathrm{AU}$ radius would then be an object of a mere 1 arcsecond in diameter on the sky. With a ground based 8-meter class telescope with adaptive optics, observing at $\lambda=3 \mu \mathrm{m}$ wavelength, and assuming one has a perfect coronograph to blend out the stray light of the star, one obtains a resolution of $\theta=1.22 \lambda / D=4.6 \times 10^{-7}$ radian, which amounts to 0.1 arcseconds, meaning that one has just 10 resolution points over the entire disk. At longer wavelengths, which is more interesting from an observational point of view because one can then observe the thermal emission from the disk itself, the resolution goes down, leaving, for instance, just 3 resolution points at $\lambda=10 \mu \mathrm{m}$. At millimeter wavelengths we could use the new Atacama Large Millimeter Array (ALMA) interferometer: With baselines of (in the future) up to $16 \mathrm{~km}$ at wavelengths down to $350 \mu \mathrm{m}$ we will obtain a maximum spatial resolution of about 0.005 arcseconds, though this resolution will presumably only be available in the final stages of ALMA construction and under exceptionally good weather and baseline projection conditions. Current resolving capabilities of ALMA (cycle 1) are around 0.1 to 0.2 arcsecond. This little exercise shows that it is extremely challenging to study substructure in protoplanetary disks. Our quest for finding and studying vortices in these disks is therefore not an easy task.

Making it even harder is the fact that vortices may not have easy to recognize signatures. The motion of the gas, detectable through the doppler shift of e.g. CO rotational lines, will not deviate dramatically from simple Kepler rotation. The vortex rotation is a subtle, subsonic motion on top of the Keplerian motion. This is difficult to detect. The molecular weight of $\mathrm{CO}\left(m_{\mathrm{CO}}=28 m_{u}\right)$ is about 12 times larger than that of the mean molecular weight of the gas $\left(\langle m\rangle \simeq 2.3 m_{u}\right)$, meaning that the thermal broadening of $\mathrm{CO}$ lines $\left(a_{\mathrm{th}}=\sqrt{2 k T / m_{\mathrm{CO}}}\right)$ is about 3.5 times narrower than the

ae-mail: dullemond@uni-heidelberg.de 
isothermal sound speed $\left(c_{s}=\sqrt{k T /\langle m\rangle}\right)$. This means that one could, if one has perfect observations and the disk is nearly edge-on, measure deviations from Kepler rotation down to about 0.4 times the isothermal sound speed. Any deviations from Kepler rotation that are substantially subsonic are thus hard to detect. A more promising way to detect anti-cyclonic vortices is simply by looking for the corresponding density enhancement (see e.g. the study by [1]).

In this brief review I will, from this point onward, focus on dust continuum observations of protoplanetary disks. It is impossible to be complete, and it is therefore unavoidable that I will make subjective choices in references.

\section{Resolved millimeter continuum observations}

Because dust opacities for small dust grains tend to be higher at optical and infrared wavelengths than at millimeter wavelengths, and protoplanetary disk models (e.g. [2], [3]) predict such disks to be very optically thick at optical and infrared wavelengths, it is easier to spot density enhancements at millimeter wavelengths. It is only relatively recently that the sensitivity and spatial resolution of millimeter continuum observations are high enough to see deviations from "standard" disk models. In a series of papers, Andrews et al. (e.g. [4]) studied the radial distribution of matter in protoplanetary disks in this way. They concluded that most disks were consistent with the simple viscous accretion+expansion scenario of Lynden-Bell \& Pringle ([6], see also [7]). These models are simple powerlaw models with an exponential cut-off at large radii, and they are solutions to the time-dependent equations of viscous evolution of accretion disks. The models fitted through the observations show that there appears to be enough matter in the planet-forming regions of these disks to create a solar system equivalent. However, it is important to remember that these observations, done with the Submillimeter Array (SMA) in Hawaii, lack the spatial resolution to make strong statements on this. Rather, they fit the emission at scales of $>20 \mathrm{AU}$ and extrapolate inward according to the Lynden-Bell \& Pringle model. With ALMA it will be possible to better resolve the inner disk regions, typically down to scales of a few AU.

Not all disks around T Tauri and Herbig Ae stars follow the simple Lynden-Bell \& Pringle model. Some disks appear to have their inner regions removed: they appear to lack dusty material inward of some radius. These are called "transition disks", because they are believed to be disks in a further stage of evolution: in a transition phase between protoplanetary disk and young planetary system. It should be kept in mind, however, that this nomenclature is based on an assumption. There is, so far, no definite proof yet that these transition disks are indeed a transition phase between disk and planets. At any rate, the relatively few known transition disks have become extremely popular targets for observations. Originally only identified by their lack of near-infrared emission (e.g. [8]), they are now routinely seen as donut-shaped objects in millimeter maps (e.g. [9], [5]). It is not clear what physical processes cause these disks to have such a huge inner hole and appear as rings on the sky. It could perhaps be an advanced stage of EUV-driven photoevaporation, following the "UV-switch" model of Clarke et al. ([10]) and subsequent papers. But it might also be caused by a massive planet that holds back material from the outer disk from entering the inner disk. However, as Zhu et al. ([11]) showed, this scenario may require more than just 1 planet, and may not explain the near-perfect absence of dusty material inside some of these transition disks. However, Rice et al. ([13]) and Zhu et al. ([12]) propose that while a planet may not be effective enough to hold back the accreting gas, it might be able to hold back the dust. This would lead to a dust-poor inner disk, which might explain these transition disks.

Millimeter observations have also found deviations from axisymmetry in some transition disks and some normal disks. The first discovered case of such an asymmetry was AB Aurigae, which appeared 
to have a huge lopsided peanut-shaped emission at 1.4 millimeter ([14]). Many of the transition disk donuts also seem display asymmetries of this kind, tentatively seen in the images of [9] and [5] mentioned above. More convincing evidence is about to be published by several teams, but at the time of this writing these publications are not yet out. Regaly et al. ([15]), however, have shown that such peanut or banana-shaped rings can be readily explained as arising from a huge Rossby-wave-induced vortex. This Rossby-wave instability can be triggered by the presence of some obstacle in the accretion flow of the gas in the disk. This obstable can be a planet or, as in Regaly's paper, a sudden jump in the viscosity coefficient, leading to a pile-up of material in a ring around the star, which, if the radial density gradients become too large, becomes Rossby unstable. While initially multiple vortices are formed, the simulations show that eventually a single huge banana-shaped vortex remains. If we allow dust to get trapped in this vortex, the contrast of millimeter continuum emission along the ring and in radial direction can even be boosted by a large factor, leading to a very pronounced banana-shaped emission region. Whether the observed banana and peanut-shaped continuum maps are indeed due to this process remains to be proven, but it is exciting that we may already be seeing the first evidence of vortices in disks.

\section{Resolved infrared scattered light observations}

Near-infrared images of protoplanetary disks show scattered light: the stellar radiation being scattered into our direction by dust grains in the surface of the disk. Using such observations to trace inhomogeneities in the mass distribution in the disk could be problematic, because we expect the disk to be optically thick at these wavelengths. We thus see only the structure of the surface layers of the disk, not necessarily the material at the midplane. However, if for some reason the disk is not so optically thick as we expected (for instance due to grain growth or simply due to lack of material in these regions), then also scattered light can give clues to the distribution of matter in the disk. In addition to that, scattered light can trace the gradient of the matter density $(d \Sigma / d r)$ in an indirect way, because a gradient of matter surface density $\Sigma$ leads to a gradient in the height of the disk, and as a result, to a change in the amount of starlight captured by the disk (a flat disk captures less starlight than a flared disk - a simple geometric argument, see [2]).

Inhomogeneities in scattered light images of protoplanetary disks have been observed already for some while. The most prominent example is the disk around AB Aurigae, which shows clumps, spirals etc. (e.g. [16], [17]), but AB Aurigae is a special case, because it is still surrounded by an infalling envelope. Very recently, however, a number of interesting images of transition disks have been obtained with the Subaru telescope. For instance, the star HD142527 displays a huge but very narrow ring of scattered light at roughly the radius where also the millimeter ring is known to exist ([18], [19]). This ring is a little bit bumpy, i.e. it is not an exact ring. It is very tempting to speculate that this ring is caused by trapped dust in the pressure trap caused by the inner hole in the gas, and that the perturbations are perhaps caused by a planet that perturbs the Keplerian motion of the dust particles.

\section{Outlook}

With ALMA now online and continuing to be expanded and improved, we can expect major new discoveries on the issue of inhomogeneities and non-axisymmetries in protoplanetary disks. However, also ALMA has its limitations. It is going to be a major challenge to observe small-scale vortices (vortices on scales of an AU or less), but when the full ALMA array is online it might just be possible to observe and study intermediate-scale (few AU) vortices. Infrared interferometry (e.g. with the 
Very Large Telescope) can in principle already reach $<1$ AU resolution, but this technique still faces the problem of having too few baselines to reconstruct detailed images. Only relatively simple geometries can, thus far, be studied and interpreted using this method. The future European Extremely Large Telescope (E-ELT, to be built in Chile) might help, increasing the angular resolution for direct imaging by a factor of 4 over the current infrared telescopes. But this requires a bit more patience. Observational studies of large scale (tens of AU) and perhaps intermediate scale (several AU) vortices, however, appear to now be on our doorstep.

\section{References}

[1] Wolf, S. \& Klahr, H., ApJ 578, L79 (2002)

[2] Chiang, E. \& Goldreich, P., ApJ 490, 368 (1997)

[3] D’Alessio, P., Canto, J., Calvet, N. \& Lizano, S., ApJ 500, 411 (1998)

[4] Andrews, S.M., Wilner, D., Hughes, A.M., Qi, C., Dullemond, C.P., ApJ 700, 1502 (2009)

[5] Andrews, S.M., Wilner, D.J., Espaillat, C., Hughes, A.M., Dullemond, C.P., McClure, M.K., Qi, C., Brown, J.M., ApJ 732, 42 (2011)

[6] Lynden-Bell, D. \& Pringle, J., MNRAS 168, 603 (1974)

[7] Hartmann, L., Calvet, N., Gullbring, E., D’Alessio, P., ApJ 495, 385 (1998)

[8] Calvet, N., D’Alessio, P., Hartmann, L., Wilner, D., Walsh, A., Sitko, M., ApJ 568, 1008 (2002)

[9] Brown, J.M., Blake, G.A., Qi, C., Dullemond, C.P., Wilner, D., Williams, J.P., ApJ 704, 496 (2009)

[10] Clarke, C.J., Gendrin, A., Sotomayor, M., MNRAS 328, 485 (2001)

[11] Zhu, Z., Nelson, R.P., Hartmann, L., Espaillat, C., Calvet, N., ApJ 729, 47 (2011)

[12] Zhu, Z., Nelson, R.P., Dong, R., Espaillat, C., Hartmann, L., ApJ 755, 6 (2012)

[13] Rice, W., Armitage, P.J., Wood, K., Lodato, G., MNRAS 373, 1619 (2006)

[14] Piétu, V., Guilloteau, S., Dutrey, A., A\&A 443, 945 (2005)

[15] Regály, Z., Juhász, A., Sándor, Z., Dullemond, C.P., MNRAS 419, 1701 (2012)

[16] Grady, C.A., Woodgate, B., Bruhweiler, F.C., Boggess, A., Plait, P., Lindler, D.J., Clampin, M., Kalas, P., ApJ 523, 151 (1999)

[17] Fukagawa, M., Hayashi, M., Tamura, M., Itoh, Y., Hayashi, S.S., Oasa, Y., Takeuchi, T., Morino, J., Murakawa, K., Oya, S., Yamashita, T., Suto, H., Mayama, S., Naoi, T., Ishii, M., Pyo, T., Nishikawa, T., Takato, N., Usuda, T., Ando, H., Iye, M., Miyama, S.M., Kaifu, N., ApJ 605, 53 (2004)

[18] Rameau, J., Chauvin, G., Lagrange, A., Thébault, P., Milli, J., Girard, J.H., Bonnefoy, M., A\&A 546, 24 (2012)

[19] Casassus, S., Perez, M.S., Jordán, A., Ménard, F., Cuadra, J., Schreiber, M.R., Hales, A.S., Ercolano, B., ApJ 754, 31 (2012) 